\title{
CFK-Bootsrumpf mit innovativer Technologie
}

Rhepro 21 steht für einen reproduzierbaren, auffaserverstärkten Kunststoffen basierenden Bootsentwurf des Segelclubs RHE in Hamburg. Assistenten des Instituts für Kunststoffverarbeitung (IKV) an der RWTH Aachen unterstützen mit dem akademischen Segelverein der RWTH (ASV) die Herstellung der Rumpf- und Deckschale der Rhepro 21. Die beiden Schalen werden vollständig in Sandwichbauweise mit kohlenstofffaserverstärkten Kunststoffen (CFK) im VAP-Verfahren hergestellt.

Bei der Fertigung der Rumpf- und Deckschale mittels dieser Technik (vacuum assisted process) erreicht das Team der Rhepro 21 in Aachen nach eigenen Angaben eine flächige und vollständige Evakuierung sowie ein optimales Infusionsergebnis ohne trockene Stellen. Der Laminataufbau der beiden Schalen basiert auf einem $10 \mathrm{~mm}$ dicken PET-Strukturschaumstoff des Typs Airex T92.100. Dieser wird eingefasst durch $1 \mathrm{~mm}$ dicke Decklaminate aus Kohlenstofffaser-Biaxialgelegen. Für die Imprägnierung von Schaum und Fasern wird das niedrigviskose Epoxidharzsystem RIM135/RIMH137 verwendet.

Neben der hohen Prozesssicherheit durch das Anwenden des VAP-Verfahrens überzeuge das Fertigungsergebnis durch sehr gute Oberflächen, Struktureigenschaften

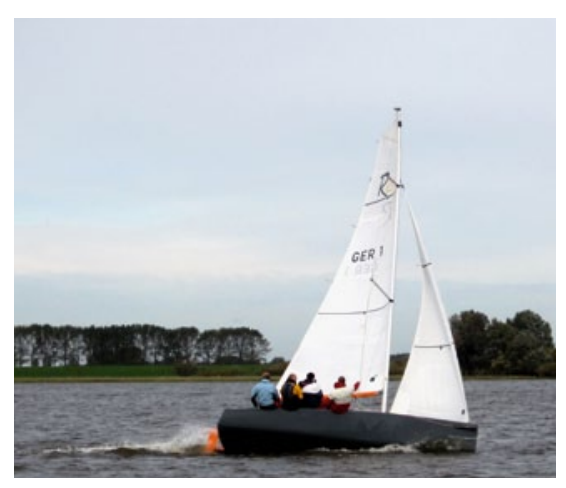

und sehr geringe Porositäten in den Bauteilen. Mit dem VAP-Membransystem der
Trans-Textil GmbH und dem verfahrenstechnischen Support der Composyst GmbH gelinge die zügige Technologieumsetzung. Die beim Segelclub RHE vorhandenen und für das Projekt kostenfrei zur Verfügung gestellten Formwerkzeuge vermieden zusätzliche Investitionen.

Die für den Eigenbau konzipierte Kieljolle ist 6,40 $\mathrm{m}$ lang, 2,40 $\mathrm{m}$ breit und besitzt eine Segelfläche von bis zu $70 \mathrm{~m}^{2}$. Der $2 \mathrm{~m}$ lange Hubkiel, den das Aachener Team beim Boot mit der Baunummer 3 erstmals in der Schwenkkiel-Technologie umsetzt, soll einen variablen Tiefgang ermöglichen. Das Gesamtgewicht des Bootes, inklusive des Kiel-Ballasts von circa 230 kg, soll nach der Fertigstellung 500 kg nicht übersteigen. Zum idealen Zielgewicht soll dem Aachener Team das VAP-Verfahren verhelfen. Dies erlaube im Vergleich zu traditionellen Infusions-Verfahren die exakte Berechnung der benötigten Harzmenge ohne ein zusätzliches Durchspülen im Fertigungsprozess.

\section{SPANTEC-LIGHT}

\section{Spantec-light}

Leichtbauwerkstoffe stehen im Mittelpunkt des neuen Forschungsverbundes Spantec-light in Baden-Württemberg. Die Wissenschaftler der Hochschulen in Aalen, Mannheim und Ulm werden künftig an Forschungsprojekten zu Werkstofftechnikund Leichtbauthemen in Kooperation zusammen arbeiten. Experten regionaler und bundesweiter Unternehmen stehen dem Hochschulverbund hierbei beratend zur Seite, darunter Carl Zeiss, Mapal, Bosch, Daimler, Eurocopter Deutschland sowie MTU Aero Engines.

Forschungsfragen des Zusammenschlusses sind die Charakterisierung von neuen Werkstoffen, wie zum Beispiel faserverstärk- ten Kunststoffen, die optimale Nutzung der spezifischen Eigenschaften, die Werkstoffherstellung, die Bearbeitungs- und Einsatzmöglichkeiten sowie die Gestaltung der Werkzeuge zur Bearbeitung der Verbundwerkstoffe.

Die Wissenschaftler der Hochschule Aalen bringen Expertise zur vollautomatisierten mikroskopischen Charakterisierung von Werkstoffen, zu zerstörungsfreien Prüf- und Messverfahren und zur Charakterisierung und simulationsgestützten Auslegung von Bauteilen mit ein. Die Hochschule UIm und die Hochschule Mannheim stellen Experten auf dem Gebiet der Zerspanung von Faserverbund- werkstoffen und der Reibungs- und Verschleißreduzierung bereit.

Unter Koordination der Hochschule Aalen wird der Forschungsverbund vom Land Baden-Württemberg für drei Jahre mit etwa 1,2 Millionen Euro gefördert. Die Förderung erfolgt im Rahmen des Programms Zentren für Angewandte Forschung an den Fachhochschulen (ZAFH). Bei dem Programm handelt es sich um landesweite Forschungsverbünde, die hochschulübergreifend und zeitlich befristet zu innovativen Forschungsthemen im Rahmen einer wettbewerblichen Ausschreibung seit 2002 in Baden-Württemberg eingerichtet und gefördert werden. 